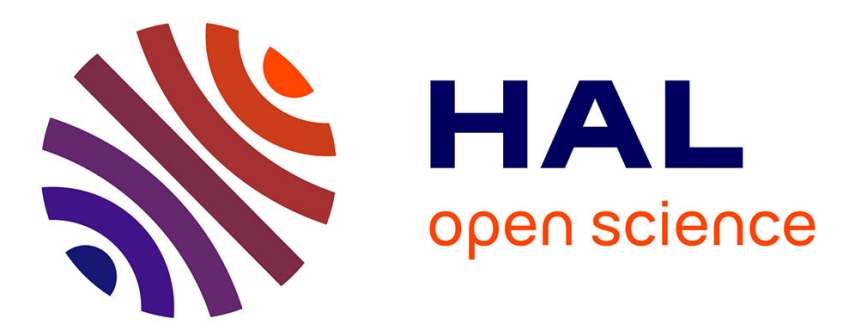

\title{
Excitation de l'azote dans une décharge microondes à fortes pressions
}

M. Locqueneux-Lefebvre, André Ricard

\section{To cite this version:}

M. Locqueneux-Lefebvre, André Ricard. Excitation de l'azote dans une décharge microondes à fortes pressions. Revue de Physique Appliquée, 1977, 12 (9), pp.1213-1217. 10.1051/rphysap:019770012090121300 . jpa-00244301

\section{HAL Id: jpa-00244301 https://hal.science/jpa-00244301}

Submitted on 1 Jan 1977

HAL is a multi-disciplinary open access archive for the deposit and dissemination of scientific research documents, whether they are published or not. The documents may come from teaching and research institutions in France or abroad, or from public or private research centers.
L'archive ouverte pluridisciplinaire HAL, est destinée au dépôt et à la diffusion de documents scientifiques de niveau recherche, publiés ou non, émanant des établissements d'enseignement et de recherche français ou étrangers, des laboratoires publics ou privés. 
Classification

Physics Abstracts

52.20

\title{
EXCITATION DE L'AZOTE DANS UNE DÉCHARGE MICROONDES A FORTES PRESSIONS (*)
}

\author{
M. LOCQUENEUX-LEFEBVRE
}

Laboratoire de Chimie Générale, Paris VI, France

et

\section{A. RICARD}

Laboratoire de Physique des Plasmas (**)

Université Paris-Sud, 91405 Orsay, France

(Reçu le 30 décembre 1976, accepté le 23 mai 1977)

\begin{abstract}
Résumé. - Un dispositif permettant de réaliser des décharges de microondes $(2,450 \mathrm{MHz})$ dans l'azote et dans l'air à pression élevée est présenté. L'étude spectroscopique de la décharge dans l'azote est faite entre 10 et 650 torr et pour des puissances UHF allant de 130 à 420 watts. L'excitation vibrationnelle, déterminée à partir du second système positif de $\mathrm{N}_{2}$, est forte correspondant à des températures vibrationnelles $T_{\mathrm{v}, x}$ de 6 à $10 \times 10^{3} \mathrm{~K}$. La comparaison avec l'état ionique, déterminée à partir du premier système négatif de $\mathbf{N}_{2}^{+}$montre que l'excitation de l'ion $\mathbf{N}_{2}^{+}$ est prédominante aux fortes pressions et fortes puissances.
\end{abstract}

Abstract. - A device generating microwave discharges in nitrogen or air is depicted. A spectroscopic study of the $\mathrm{N}_{2}$ discharge has been made for a range of pressures from 10 to 650 torr and UHF power from 130 to 420 watts. The vibrational excitation as measured from the $\mathrm{N}_{2}$ positive second system is high corresponding to a vibrational temperature $T_{\mathrm{v}, x}$ from 6 to $10 \times 10^{3} \mathrm{~K}$. The comparison with the ionic state as measured from the $\mathrm{N}_{2}^{+}$negative first system shows that the ion $\mathrm{N}_{2}^{+}$excitation is predominant for high pressures and high power levels.

1. Introduction. - Les décharges électriques dans les gaz constituent des sources privilégiées d'espèces énergétiques utilisables pour réaliser des synthèses en chimie des plasmas. Les décharges U. H. F. nous ont paru particulièrement favorables à l'étude de telles réactions. En effet, nous avons montré [1] qu'avec une décharge de $2,450 \mathrm{MHz}$, on peut exciter l'azote et l'air jusqu'à la pression atmosphérique.

Les bilans d'excitation, d'ionisation et de dissociation constituent alors des données fondamentales pour la réalisation de telles synthèses. Ainsi, les rendements en oxydes d'azote à partir des mélanges $\mathrm{N}_{2}-\mathrm{O}_{2}$ vont dépendre des proportions d'ions $\mathrm{N}_{2}^{+}$, de molécules $\mathrm{N}_{2}^{*}$ et d'atomes $\mathbf{N}$ présents dans le plasma.

Le spectre optique des décharges est caractéristique des premier et second systèmes positifs de $\mathrm{N}_{2}$ et du premier système négatif de $\mathrm{N}_{2}^{+}$.

Dans ce travail, nous précisons l'état d'excitation de

(*) Communication présentée au Congrès National de Physique des Plasmas, Paris, 6-10 décembre 1976.

(**) Laboratoire associé au C. N. R. S. l'azote dans la décharge hyperfréquence pour des puissances comprises entre 130 et 420 watts et des pressions variant de 10 torr à 1 atmosphère. Nous considérons en particulier l'état d'excitation vibrationnelle de $\mathrm{N}_{2}$ et comparons l'excitation de l'ion $\mathrm{N}_{2}^{+}$et de la molécule $\mathrm{N}_{2}$ en fonction de la pression et de la puissance de la décharge.

2. Dispositif expérimental. - Les plasmas sont produits en système dynamique dans un tube en silice transparente (diamètre intérieur $10 \mathrm{~mm}$ ) traversant, comme indiqué sur figure 1 , une cavité résonnante construite suivant le modèle de la cavité $\mathrm{n}^{\circ} 1$ de Goudmand et al. [2]. Cette cavité est alimentée par une source $2,450 \mathrm{MHz}$ (Thomson C. S. F.) qui fournit une puissance que l'on peut faire varier entre 15 et 1500 watts. La transmission de l'onde à la cavité se fait par l'intermédiaire d'un câble coaxial. Un isolateur (Marconi) protège le magnétron contre les ondes réfléchies.

La puissance incidente et la puissance réfléchie sont mesurées par un coupleur bidirectif $(60 \mathrm{~dB})$ situé entre l'isolateur et le câble coaxial. Dans ce but, on utilise 


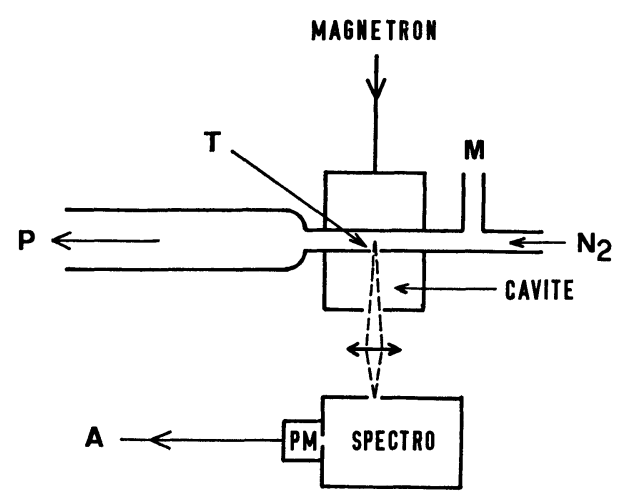

Fig. 1. - Schéma de l'appareil. $T$ : réacteur en silice, $P$ : pompe à vide, $\mathrm{M}$ : manomètre à mercure, $\mathrm{P}$. $\mathrm{M}$. : Photomultiplicateur, A : amplificateur de courant et enregistreur.

[Apparatus diagram. $\mathrm{T}$ : quartz reactor, $\mathbf{P}$ : vacuum pump, M : mercury manometer, P. M. : Photomultiplier, A : current amplifier and trace-recorder.]

une thermistance $478 \mathrm{~A}$ et un multiwattmètre (HewlettPackard).

La pression et le débit dans le réacteur sont réglés par une pompe de $20 \mathrm{~m}^{3} / \mathrm{h}$ et des robinets à pointeaux. Ils sont mesurés juste avant la cavité par un manomètre à mercure et des débitmètres Brooks. Le gaz utilisé est de l'azote $\mathrm{R}$ de l'Air Liquide.

La cavité a été percée à la hauteur de l'antenne d'une fente rectangulaire de $2 \mathrm{~mm}$ de largeur et $3,5 \mathrm{~mm}$ de hauteur pour permettre l'observation spectroscopique de la décharge à haute pression. Les spectres d'émission sont enregistrés entre 3000 et $9000 \AA$ à l'aide d'un spectromètre Jobin-Yvon HRS 1 avec une résolution $\delta \lambda=3$ à $4 \AA$. Le monochromateur est équipé d'un photomultiplicateur RCA C 31. Le signal de sortie du PM est amplifié à l'aide d'un picoampèremètre Keithley $414 \mathrm{~S}$ et enregistré.

Nous avons opéré dans les conditions suivantes:

\begin{tabular}{cl|c}
$\begin{array}{c}\text { Pressions } \\
\text { torr }\end{array}$ & $\begin{array}{c}\text { Débit } \\
\text { l/min. }\end{array}$ & $\begin{array}{c}\text { Puissance } \\
\text { U. H. F. } \\
\text { watts }\end{array}$ \\
10 & \multicolumn{1}{c}{-} & -130 \\
25 & 5 et 1 & 145 \\
50 & 1 & 185 \\
100 & 1 et 0,25 & 215 \\
300 & 1 et 0,25 & 345 \\
450 & 0,25 & 420 \\
642,5 & 0,25 &
\end{tabular}

Le plasma est inhomogène aux pressions $p>25$ torr. Les observations effectuées sont relatives à la zone ionisée qui se trouve face à l'antenne d'excitation.

3. Excitation vibrationnelle de l'azote. - Pour des pressions inférieures à 100 torr, le second système positif de l'azote $\left(\mathrm{C}^{3} \Pi_{\mathrm{u}}, v^{\prime} \rightarrow \mathrm{B}^{3} \Pi_{\mathrm{g}}, v^{\prime \prime}\right)$ est émis avec une intensité suffisante pour permettre la mesure de l'excitation vibrationnelle. En effet, les intensités $I\left(v^{\prime}\right.$, $\left.v^{\prime \prime}\right)$ des têtes de bande de la séquence $v^{\prime}-v^{\prime \prime}=-2$ sont reliées aux populations $N\left(C, v^{\prime}\right)$ des quatre niveaux vibrationnels de l'état $\mathrm{C}^{3} \Pi_{\mathrm{u}}$ par la relation :

$$
I\left(v^{\prime}, v^{\prime \prime}\right)=C(\lambda) N\left(C, v^{\prime}\right) \frac{q\left(v^{\prime}, v^{\prime \prime}\right)}{\lambda^{3}\left(v^{\prime}, v^{\prime \prime}\right)}
$$

où $C(\lambda)$ est une constante qui dépend du volume émissif et de la réponse spectrale du système de mesure, $q\left(v^{\prime}\right.$, $\left.v^{\prime \prime}\right)$ sont les facteurs de Franck-Condon pour lesquels nous avons choisi les valeurs calculées par Mc Callum et al. [3] et mesurées par Shemansky et Broadfoot [4]. Les facteurs de Franck-Condon sont liés à la probabilité radiative d'Einstein par la relation:

$$
A\left(v^{\prime}, v^{\prime \prime}\right)=\frac{q\left(v^{\prime}, v^{\prime \prime}\right)}{\lambda^{3}\left(v^{\prime}, v^{\prime \prime}\right)}
$$

Les populations $N\left(C, v^{\prime}\right)$ sont reportées en coordonnées semi-logarithmiques sur la figure 2 en normalisant

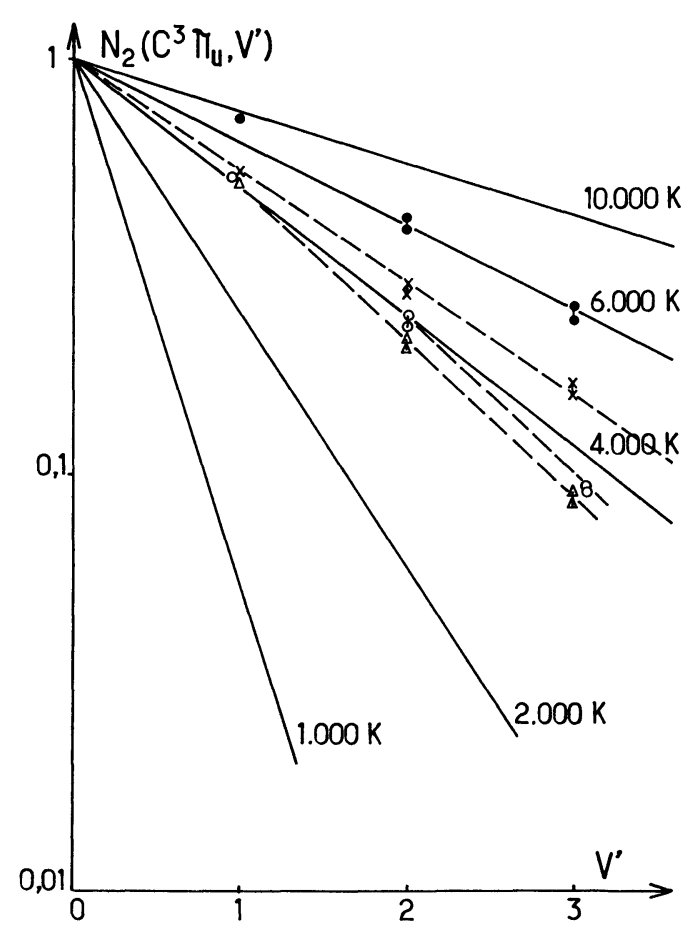

FIG. 2. - Détermination des températures vibrationnelles apparentes $T_{\mathrm{v}}^{\prime} . \quad P_{\mathrm{UHF}}=130$ watts, $D=1 \mathrm{l} / \mathrm{min} ., \quad P=25 \mathrm{~T}(\times)$, $50 \mathrm{~T}(\bigcirc)$, et $100 \mathrm{~T}(\triangle), D=5 \mathrm{l} / \mathrm{min} ., P=10 \mathrm{~T}(\bullet)$.

[Formal vibrational temperature $T_{\mathrm{v}}^{\prime}$ determination. $P_{\mathrm{UHF}}=$ 130 watts, nitrogen flow $=1 \mathrm{l} / \mathrm{min} ., \quad P=25 \mathrm{~T}(\times), 50 \mathrm{~T}(\mathrm{O})$ and $100 \mathrm{~T}(\triangle)$, nitrogen flow $=5 \mathrm{l} / \mathrm{min} ., \quad P=10 \mathrm{~T}(\bullet)$.]

à 1 la densité volumique du niveau $v^{\prime}=0$. Les droites indiquent les températures vibrationnelles apparentes $T_{\mathrm{v}}^{\prime}$ correspondant à une distribution de Boltzman des concentrations $\mathrm{N}_{2}\left(\mathrm{C}^{3} \Pi_{\mathrm{u}}, v^{\prime}\right)$ selon la relation :

$$
\frac{N\left(C, v^{\prime}\right)}{N(C, 0)}=\exp -\left(\frac{\Delta E_{0}^{v^{\prime}}}{k T_{v}^{\prime}}\right)
$$

où $\Delta E_{0}^{v^{\prime}}$ est l'écart énergétique entre les niveaux vibrationnels $v^{\prime}$ et 0 et $k$ la constante de Boltzman. 
Cette température apparente reflète l'action des collisions électroniques et des particules lourdes qui excitent et désexcitent l'état $\mathrm{C}^{3} \Pi_{\mathrm{u}}$.

On constate sur la figure 2 que, pour les faibles puissances U. H. F. (130 watts) la température apparente diminue légèrement $\left(T_{\mathrm{v}}^{\prime}\right.$ passe de $\left.6 \mathrm{à} 4 \times 10^{3} \mathrm{~K}\right)$ lorsque la pression augmente de 10 à 100 torr. Aux fortes puissances (420 watts), $T_{\mathrm{v}}^{\prime}$ ne varie pratiquement pas.

Nous avons cherché à déterminer la température de vibration vraie de l'azote $T_{\mathrm{v}, X}$ en considérant que les niveaux $\left(\mathrm{C}^{3} \Pi_{\mathrm{u}}, v^{\prime}\right)$ sont peuplés et détruits selon les processus suivants :

1) excitation par collisions électroniques directes à partir du fondamental $X^{1} \Sigma_{\mathrm{g}}^{+}$, de population $N\left(X, v^{\prime \prime \prime}\right)$;

2) désexcitation radiative ;

3) relaxation par collisions avec les neutres.

Suivant le processus 1 , le taux d'excitation du niveau $\left(\mathrm{C}^{3} \Pi_{\mathrm{u}}, v^{\prime}\right)$ peut s'écrire :

$$
\left[\frac{\mathrm{d} N\left(C, v^{\prime}\right)}{\mathrm{d} t}\right]_{1}=C\left(n_{\mathrm{e}}, T_{\mathrm{e}}\right) \sum_{v^{\prime \prime \prime}} N\left(X, v^{\prime \prime \prime}\right) q\left(v^{\prime \prime \prime}, v^{\prime}\right)
$$

où $C\left(n_{\mathrm{e}}, T_{\mathrm{e}}\right)$ est un coefficient qui dépend des concentrations et des températures électroniques et $q\left(v^{\prime \prime \prime}, v^{\prime}\right)$ sont les facteurs de Franck-Condon pour le transfert considéré.

Les taux de désexcitation suivant les processus 2 et 3 s'écrivent :

$$
\begin{aligned}
& {\left[\frac{\mathrm{d} N\left(C, v^{\prime}\right)}{\mathrm{d} t}\right]_{2,3}=} \\
& \quad=-N\left(C, v^{\prime}\right)\left[\sum_{v^{\prime \prime}} A\left(v^{\prime}, v^{\prime \prime}\right)+N(X) Q_{\mathrm{c}}\left(v^{\prime}\right)\right]
\end{aligned}
$$

où $\sum_{v^{\prime \prime}} A\left(v^{\prime}, v^{\prime \prime}\right)$ est la probabilité d'émission radiative du niveau $C$, $v^{\prime}$ et $Q_{\mathrm{c}}\left(v^{\prime}\right)$ est le coefficient de relaxation avec les neutres.

A l'équilibre :

$$
\left[\frac{\mathrm{d} N\left(C, v^{\prime}\right)}{\mathrm{d} t}\right]_{1}=\left[\frac{\mathrm{d} N\left(C, v^{\prime}\right)}{\mathrm{d} t}\right]_{2,3}
$$

et

$$
N\left(C, v^{\prime}\right)=C\left(n_{\mathrm{e}}, T_{\mathrm{e}}\right) \frac{\sum_{v^{\prime \prime \prime}} N\left(X, v^{\prime \prime \prime}\right) q\left(v^{\prime \prime \prime}, v^{\prime}\right)}{\alpha\left(C, v^{\prime}\right) \sum_{v^{\prime \prime}} A\left(v^{\prime}, v^{\prime \prime}\right)}
$$

avec

$$
\alpha\left(C, v^{\prime}\right)=1+\frac{N(X) Q_{c}\left(v^{\prime}\right)}{\sum_{v^{\prime \prime}} A\left(v^{\prime}, v^{\prime \prime}\right)}
$$

Nous utilisons les valeurs de la réf. [5] pour le calcul de $q\left(v^{\prime \prime \prime}, v^{\prime}\right)$, celles de la réf. [6] pour $\sum_{v^{\prime \prime}} A\left(v^{\prime}, v^{\prime \prime}\right)$ et celles des réfs. [7] et [8] pour $Q_{\mathrm{c}}\left(v^{\prime}\right)$.

On obtient ainsi pour $\alpha\left(C, v^{\prime}\right)$ les valeurs suivantes :

$$
\begin{aligned}
& \alpha(C, 0)=1+1,8 \times 10^{-2} \mathrm{P} ; \\
& \alpha(C, 1)=1+(1,9-5,6) \times 10^{-2} \mathrm{P} ; \\
& \alpha(C, 2)=\alpha(C, 3)=1+(3,8-6,3) \times 10^{-2} \mathrm{P} .
\end{aligned}
$$

Les deux bornes indiquées sur les valeurs de $\alpha(C, 1,2$ et 3) sont dues à la dispersion des résultats des réfs. [7] et [8].

En écrivant la relation (4), nous ne tenons pas compte de la relaxation vibrationnelle suivant le processus : $C, v^{\prime}+X, v^{\prime \prime} \rightarrow C, v^{\prime}-1+X, v^{\prime \prime}+1$, introduit dans la réf. [8] mais non observé dans une publication récente [13]. De même, nous négligeons aux fortes pressions utilisées ici l'effet des collisions électroniques.

La relation (4) permet en principe de déterminer la distribution des niveaux vibrationnels $N\left(X, v^{\prime \prime \prime}\right)$ à partir des valeurs expérimentales $N\left(C, v^{\prime}\right)$. En fait, les imprécisions sur les coefficients rendent cette détermination illusoire. Aussi, nous avons supposé que les niveaux vibrationnels de l'état fondamental $X^{\mathbf{1}} \Sigma_{\mathrm{g}}^{+}$se répartissent suivant la distribution de Boltzman :

$$
\frac{N\left(X, v^{\prime \prime \prime}\right)}{N(X, 0)}=\exp -\left(\frac{\Delta \mathrm{E}_{0}^{v^{\prime \prime \prime}}}{k T_{\mathrm{v}, X}}\right) \text {. }
$$

D'une manière analogue à la figure 2 , nous avons tracé (Fig. 3) les courbes $N\left(C, v^{\prime}\right)=f\left(v^{\prime}\right)$ pour plusieurs valeurs de $T_{\mathrm{v}, X}$. Un tel réseau de courbes doit

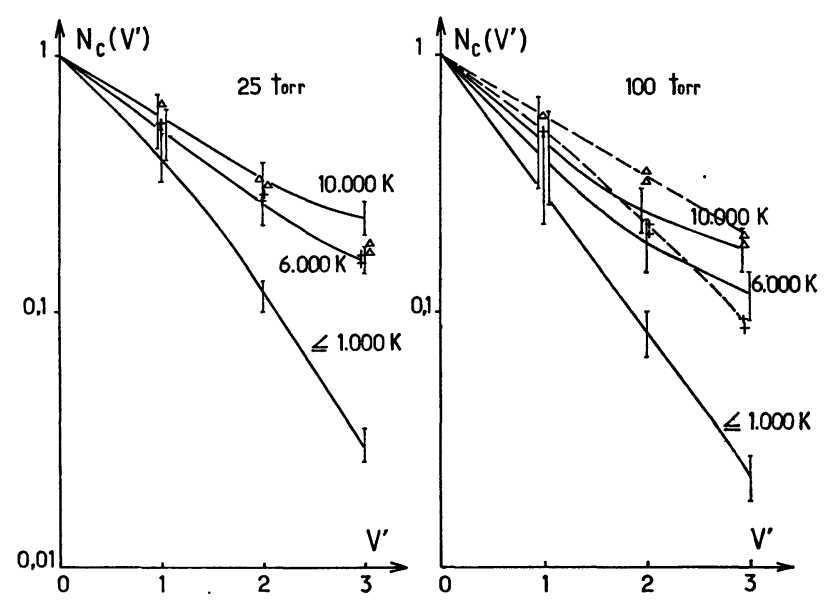

FIG. 3. - Détermination des températures vibrationnelles vraies $T_{\mathrm{v}, X} \cdot P_{\mathrm{UHF}}=130 \mathrm{~W}(\times), 420 \mathrm{~W}(\triangle), P=25$ et $100 T$, $D=11 / \mathrm{min}$.

[True vibrational temperature $T_{\mathrm{v}, X}$ determination. $P_{\mathrm{UHF}}=130 \mathrm{~W}(\times), 420 \mathrm{~W}(\Delta)$, $P=25$ and $100 \mathrm{~T}$, nitrogen flow $=1 \mathrm{l} / \mathrm{min}$.]

être tracé pour chaque pression d'azote. Les courbes de la figure 3 correspondent à $P=25$ torr et $P=100$ torr, les barres représentent les bornes de $\alpha$.

Les valeurs expérimentales sont reportées pour deux puissances U. H. F. : (+) 130 watts et $(\Delta) 420$ watts. A l'exception des résultats obtenus à forte puissance à 100 torr, les points expérimentaux se distribuent entre les valeurs 6 et $10 \times 10^{3} \mathrm{~K}$ de $T_{\mathrm{v}, x}$.

Ces valeurs de $T_{v, X}$ sont compatibles avec celles obtenues pour $T_{\mathrm{v}}^{\prime}$. La différence entre les résultats obtenus est en effet due aux phénomènes de relaxation qui, comme on peut le voir d'après $\alpha\left(C, v^{\prime}\right)$, augmentent avec $v^{\prime}$. Cette similarité des valeurs de $T_{\mathrm{v}, X}$ et $T_{\mathrm{v}}^{\prime}$ est en 
TABLEAU I

Facteurs Franck-Condon pour les transitions $A^{3} \Sigma_{\mathrm{u}}^{+}, 0-1 \rightarrow C^{3} \Pi_{\mathrm{u}}, v^{\prime}$ suivant $R$. W. Nicholls [11]. $(-01)=10^{-1}$ [Franck-Condon factors for the $A^{3} \Sigma_{\mathrm{u}}^{+}, 0-1 \rightarrow C^{3} \Pi_{\mathrm{u}}, v^{\prime}$ transitions, from the R. W. Nicholls [11] data. $(-01)=10^{-1}$.]

$$
\begin{array}{ccc}
A^{3} \Sigma_{\mathbf{u}}^{+}, v^{\prime \prime} & \frac{0}{-} & \frac{1}{0} \\
1 & 0,330(-01) & 0,139(00) \\
1 & 0,920(-01) & 0,189(00)
\end{array}
$$

$$
C^{3} \Pi_{\mathrm{u}}, v^{\prime}
$$

soi une confirmation du modèle choisi pour établir la relation (4).

Nous voulons cependant discuter la première hypothèse en envisageant une excitation de l'état $\mathrm{C}^{3} \Pi_{\mathrm{u}}$ en deux étapes par l'intermédiaire de l'état $\mathrm{A}^{3} \Sigma_{\mathrm{u}}^{+}$.

Nous avons considéré que l'état $A^{3} \Sigma_{\mathrm{u}}^{+}$se trouve, en raison d'une rapide relaxation vibrationnelle $[9,10]$, principalement excité sur les niveaux 0 et 1 . La distribution vibrationnelle de l'état $C^{3} \Pi_{\mathrm{u}}$, produite par collisions électroniques suivant les étapes : $A^{3} \Sigma_{\mathrm{u}}^{+}$, $0-1 \rightarrow C^{3} \Pi_{u}, v^{\prime}$, est alors calculée à partir de la relation suivante :

$$
\begin{aligned}
\alpha\left(C, v^{\prime}\right) \sum_{v^{\prime \prime}} A\left(v^{\prime}, v^{\prime \prime}\right) & \frac{N\left(C, v^{\prime}\right)}{N(A, 0)}= \\
& =C\left(n_{\mathrm{e}}, T_{\mathrm{e}}\right)\left[q\left(0, v^{\prime}\right)+q\left(1, v^{\prime}\right)\right]
\end{aligned}
$$

où nous avons pris $N(A, 0) \sim N(A, 1)$, ce qui est admis comme étape finale de la relaxation vibrationnelle des niveaux $A^{3} \Sigma_{\mathrm{u}}^{+}$[9].

Les facteurs de Franck-Condon pour la transition $A^{3} \Sigma_{\mathrm{u}}^{+}, \quad 0-1 \rightarrow C^{3} \Pi_{\mathrm{u}}, v^{\prime}$ ont été calculés par R. W. Nicholls [11] à partir d'un potentiel Klein-Dunham. Les résultats de ce calcul sont reportés dans le tableau I. Dans le tableau II, nous indiquons la distri-

\section{TABLEAU II}

Distribution vibrationnelle de l'état $C^{3} \Pi_{\mathrm{u}}, v^{\prime}$ suivant

\begin{tabular}{|c|c|c|c|c|c|c|}
\hline$v^{\prime}$ & & 0 & 1 & 2 & 3 & 4 \\
\hline & $10 \mathrm{~T}$ & 1 & 2,1 & 1,9 & 1,5 & 1,6 \\
\hline & $20 \mathrm{~T}$ & 1 & 1,9 & 1,6 & 1,2 & 1,4 \\
\hline$N\left(C^{3} \Pi_{\mathrm{u}}\right)$ & $50 \mathrm{~T}$ & 1 & 1,6 & 1,2 & 0,9 & 1,1 \\
\hline & $100 \mathrm{~T}$ & 1 & 1,4 & 1 & 0,8 & 0,9 \\
\hline
\end{tabular}
l'excitation $A^{3} \Sigma_{\mathrm{u}}^{+}, v_{4}=0-1 \rightarrow C^{3} \Pi_{\mathrm{u}}, v^{\prime}$. Normalisation : $N(C, 0)=1$.

[Vibrational distribution of the $C^{3} \Pi_{\mathrm{u}}, v^{\prime}$ state following the excitation $A^{3} \Sigma_{\mathrm{u}}^{+}, v_{4}=0-1 \rightarrow C^{3} \Pi_{\mathrm{u}}, v^{\prime}$. Normalisation : $N(C, 0)=1$.]

bution vibrationnelle de $N_{2}\left(C^{3} \Pi_{\mathrm{u}}, v^{\prime}\right)$ calculée suivant l'équation (6) pour des pressions d'azote comprises entre 10 et 100 torr. Nous avons appliqué la normalisation $N(C, 0)=1$. On constate qu'une excitation en deux étapes conduirait à une surpopulation des états $C^{3} \Pi_{\mathrm{u}}, v^{\prime} \geqslant 1$. Les résultats expérimentaux reportés sur la figure 3 ne montrent pas cet effet. L'hypothèse d'une excitation directe se trouve ainsi renforcée.

4. Excitation de l'ion $\mathbf{N}_{2}^{+}$. - Quand on fait croître la pression, on constate que les raies spectrales des $1^{\text {er }}$ et $2^{\mathrm{e}}$ systèmes positifs de l'azote diminuent en intensité, à l'inverse des raies ioniques du $1{ }^{\text {er }}$ système négatif dont l'intensité augmente et ceci d'autant plus que la puissance U. H. F. est élevée. Ainsi pour $P>300$ torr, le spectre dans le visible ne comporte que les raies du $1^{\text {er }}$ système négatif.

Nous avons choisi d'étudier les variations du rapport $r$ des intensités des raies ioniques $\lambda=3914 \AA$ et neutre $\lambda=3805 \AA$ avec la pression pour un débit de gaz constant. Les intensités des raies ont été mesurées au maximum de la tête de bande qui, pour ces deux raies, est constitué par la branche rotationnelle $P$.

Les résultats obtenus sont représentés sur la figure 4. On constate que le rapport $r$ croît (surtout au-dessus de. 50 torr) avec la pression et la puissance de la décharge. Sur cette figure, nous avons aussi tracé la variation du rapport

$$
r_{\mathrm{Q}}=\frac{\alpha(C, 0)}{\alpha\left(B^{+}, 0\right)}
$$

avec la pression dans lequel $\alpha(C, 0)$ et $\alpha\left(B^{+}, 0\right)$ exprimés par la relation (5) caractérisent la relaxation des niveaux $v^{\prime}=0$ des états neutre $N_{2}\left(C^{3} \Pi_{u}\right)$ et ionique $N_{2}^{+}\left(B^{2} \Sigma_{\mathrm{u}}^{+}\right)$. Les valeurs de $\alpha$ ont été calculées à partir des coefficients de relaxation publiés dans les réf. [6] à [8].

On constate que les rapports d'intensité

$$
r=\frac{I(3914 \AA)}{I(3805 \AA)}
$$

varient en sens inverse de $r_{\mathrm{Q}}$. Les variations des valeurs expérimentales ne correspondent donc pas uniquement à un effet de relaxation avec les neutres. De même, le calcul du rapport $r$, dans l'hypothèse d'un équilibre thermodynamique local (E. T. L.) [12], donne des valeurs de

$$
r=\frac{I(3914 \AA)}{I(3805 \AA)}
$$




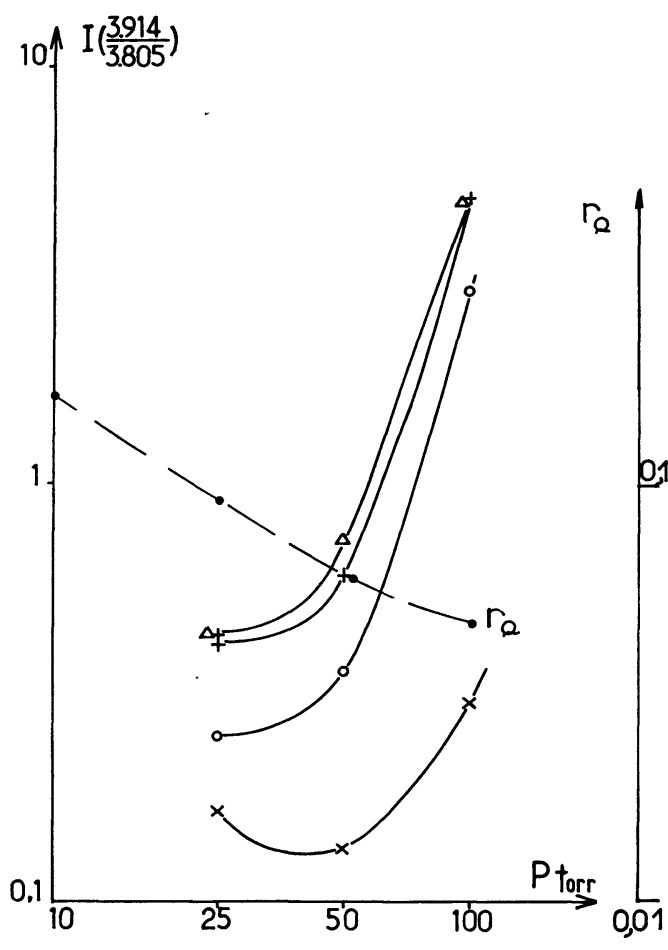

FIG. 4. - Variation du rapport $r$ des intensités des raies ioniques $\lambda=3,914 \AA$ et neutre $\lambda=3,805 \AA$ avec la pression. Comparaison avec la valeur théorique $r_{\mathrm{Q}} . D=1 \mathrm{l} / \mathrm{min}$., $P_{\mathrm{UHF}}=130 \mathrm{~W}(\times)$, $215 \mathrm{~W}(O), 345 \mathrm{~W}(+)$ et $420 \mathrm{~W}(\Delta)$

[Variation of the ratio $(r)$ of the ionic line $(\lambda=3.914 \AA)$ and neutral line $(\lambda=3.805 \AA)$ versus pressure. Comparison with theoretical value $r_{Q}$. nitrogen flow $=11 / \mathrm{min} ., P_{\text {UHF }}=130 \mathrm{~W}(X)$, $215 \mathrm{~W}(O), 345 \mathrm{~W}(+)$ and $420 \mathrm{~W}(\Delta)$. comprises entre 50 et 200 à $P=100$ torr et à des températures variant de 3 à $12 \times 10^{3} \mathrm{~K}$. Les résultats expérimentaux sont bien en dessous de ces valeurs. Aux pressions $P \leqslant 100$ torr et $P_{\mathrm{UHF}} \leqslant 420$ watts, l'E. T. L. n'est certainement pas atteint.

5. Conclusion. - L'étude spectroscopique d'une décharge U. H. F. à forte pression d'azote (10-650 torr) a permis de montrer que l'excitation vibrationnelle de la molécule est élevée, correspondant à des températures $T_{\mathrm{v}, X}$ allant de 6 à $10 \times 10^{3} \mathrm{~K}$. La distribution vibrationnelle de l'état $C^{3} \Pi_{\mathrm{u}}$ est caractéristique d'une excitation par collisions électroniques directes à partir du fondamental $X^{1} \Sigma_{\mathrm{g}}^{+}$

L'excitation des raies ioniques du $1^{\mathrm{er}}$ système négatif est prédominante aux fortes pressions $(P>50$ torr) et fortes puissances U. H. F. ( $W>300$ watts).

Nous nous proposons de poursuivre ce travail en mesurant d'une part le rapport

$$
r=\frac{I(3914 \AA)}{I(3371 \AA)}
$$

pour des pressions supérieures à 100 torr (la raie $3,371 \AA$ est plus intense que la raie $3,805 \AA$ utilisée jusqu'à présent) et d'autre part, la température rotationnelle à partir de la structure des raies $\lambda=3,914 \AA$ et $\lambda=3,371 \AA$ à haute résolution $(\delta \lambda \sim 0,5 \AA)$.

Les résultats de ces mesures devraient permettre de déterminer dans quelles conditions le plasma hyperfréquence s'approche de l'E. T. L.

\section{Bibliographie}

[1] Locgueneux-Lefebvre, M. et Ben-Aim, R., C. $R$. Hebd. Séan. Acad. Sci. 282 (1976) C-97.

[2] Dupret, C., Vidal, B., Goudmand, P., Revue Phys. Appl. 5 (1970) 337.

[3] Mc Callum, Jarmain, W. R., Nicholls, R. W., CRESS Spectroscopic Report $n^{\circ} 3$ (1972).

[4] Shemansky, D. E., Broadfoot, A. L., J. Quant. Rad. Spect. Transf. 11 (1971) 1385.

[5] BenesCH, W. et al., Astrophys. J. 143 (1966) 236.
[6] Johnson, A. W., Fowler, R. G., J. Chem. Phys. 53 (1970) 65.

[7] Millet, P. et al., J. Chem. Phys. 58 (1973) 5839.

[8] Calo, J. M., Axtman, R. C., J. Chem. Phys. 54 (1971) 1332.

[9] MeYer, J. A. et al., J. Chem. Phys. 55 (1971) 2084.

[10] Dreyer, J. W., Perner, D., J. Chem. Phys. 58 (1973) 1195.

[11] Nicholls R. W. (Communication privée) (1977).

[12] Baronnet, J. M. et al., J. Physique Colloq. 34 (1973) C 2129.

[13] Chen, C. M., J. Chem. Phys. 65 (1976) 3863. 